\title{
Sub-ångstrom Experimental Validation of Molecular Dynamics for Predictive Modeling of Extended Defect Structures in Si
}

\author{
K. J. Dudeck, ${ }^{1, *}$ L. A. Marqués, ${ }^{2}$ A. P. Knights, ${ }^{3}$ R. M. Gwilliam, ${ }^{4}$ and G. A. Botton ${ }^{1, \dagger}$ \\ ${ }^{1}$ Department of Materials Science and Engineering, McMaster University, \\ 1280 Main Street West, Hamilton, Ontario L8S 4L7, Canada \\ ${ }^{2}$ Departamento de Electrónica, E.T.S.I. de Telecomunicación, Universidad de Valladolid, 47011 Valladolid, Spain \\ ${ }^{3}$ Department of Engineering Physics, McMaster University, 1280 Main Street West, Hamilton, Ontario L8S 4L7, Canada \\ ${ }^{4}$ Surrey Ion Beam Centre, University of Surrey, Guildford, Surrey GU2 7XH, United Kingdom
}

(Received 17 January 2013; published 18 April 2013)

\begin{abstract}
In this Letter we present the detailed, quantitative comparison between experimentally and theoretically derived structures of the extended $\{311\}$ defect in silicon. Agreement between experimental and theoretical column positions of better than $\pm 0.05 \mathrm{~nm}$ has been achieved for all 100 atomic columns in the defect structure. This represents a calculated density of $5.5 \times 10^{14}$ silicon interstitials per $\mathrm{cm}^{2}$ on $\{311\}$ planes, in agreement with previous work [S. Takeda, Jpn. J. Appl. Phys., Part 2, 30, L639 (1991)]. We show that although the $\{311\}$ defect is made up of five-, six-, seven-, and eight-member rings, the shape of these rings varies as a function of position along the defect, and these variations can be determined experimentally with high precision and accuracy. The excellent agreement between the calculated and experimentally derived structure, including the position of atomic columns and the shape of the distinct structural units of the defect, provides strong evidence for the quality and robustness of the molecular dynamics simulation approach for structural studies of defects. The experimental approach is straightforward, without the need for complicated image processing methods, and is therefore widely applicable.
\end{abstract}

DOI: 10.1103/PhysRevLett.110.166102

PACS numbers: 61.72.Nn, 61.43.Bn, 68.37.Ma

Predictive atomistic modeling in the microelectronics industry is becoming essential in order to reduce development times and costs [1]. To be predictive, modeling and simulation must be physically based and experimentally verified to ensure that the predicted structure is physically valid. Quantitative, high resolution electron microscopy techniques are an ideal means for providing such comparative data. However, large defects having limited symmetry present considerable challenges for quantitative electron microscopy. Imaging such defects requires high stability (e.g., limited beam and specimen drift, controlled microscope environment) in order for images to reliably reproduce the defect structure. Further, without the symmetry of defects such as high-angle $(\Sigma)$ grain boundaries and periodic interfaces, techniques such as averaging column positions within repeat units cannot be employed to increase the signal-to-noise ratio of images or reduce the experimental uncertainty of column positions [2,3]. Combined, these factors make extended structures such as the extended $\{311\}$ defect a challenging case study to extend the limits of precision and accuracy of quantitative electron microscopy.

Ion implantation followed by annealing is a wellestablished route for fabricating $p-n$ junctions in $\mathrm{Si}$ and Ge device technology. In addition to creating a distribution of dopants, this process is known to generate excess $\mathrm{Si}$ interstitials, which tend to coalesce and form extended defects $[4,5]$. These defects have been shown to have a $\{311\}$ habit plane and to be extended in the $\langle 011\rangle$ direction [6]. The number and length of these extended $\{311\}$ defects have been used to assess the concentration of Si interstitials generated after ion implantation, imperative for the modeling of the transient enhanced diffusion (TED) of interstitial diffusers such as boron. This led to the development of the " +1 " model, which states that the concentration of $\mathrm{Si}$ interstitials remaining after ion implantation and annnealing is equal to the concentration of the implanted ions [5]. This defect structure has thus proven to be of significant technological importance. In the 1990s, Takeda and colleagues studied the structure of the extended $\{311\}$ defect using high resolution transmission electron microscopy (HRTEM) and diffraction techniques and proposed an experimentally derived structural model based on qualitative comparison with the HRTEM data [6,7]. More recently, several groups have performed theoretical studies of the structure, strain, and energetics associated with the defect [7-13]. However, significant experimental investigations of the structure of the $\{311\}$ defect have remained scarce since the work of Takeda and colleagues.

In this Letter we demonstrate an agreement of better than $\pm 0.05 \mathrm{~nm}$ between atomic column positions in the theoretically predicted and experimentally observed structure of the extended $\{311\}$ defect in Si without making use of any image processing techniques, which may introduce bias or require extensive computational power. This work validates the use of the molecular dynamics (MD) simulation for predictive modeling of defect structures in Si and demonstrates that unprocessed high-angle annular darkfield (HAADF) scanning transmission electron microscopy 
(STEM) images can be used for high-precision, quantitative structural analysis.

Experimental samples were prepared by implanting $1 \times 10^{15} \mathrm{~cm}^{-3}$ indium ions into $n$-type (100) $\mathrm{Si}$ with an energy of $40 \mathrm{keV}$ and an angle of $7^{\circ}$ to the surface normal. Such conditions produce an amorphous layer approximately $50 \mathrm{~nm}$ thick. Following implantation, samples were annealed at $600{ }^{\circ} \mathrm{C}$ for $2 \mathrm{~min}$ in a $\mathrm{N}_{2}$ atmosphere using a rapid thermal annealing process. This resulted in the formation of end-of-range defects at a depth commensurate with the amorphous-crystalline interface. Imaging was performed using a FEI-Titan 80-300 STEM operated at $200 \mathrm{keV}$ and equipped with two aberration correctors. The specimens were prepared by tripod polishing combined with low voltage $(<800 \mathrm{~V})$ ion milling. A convergence angle of $19 \mathrm{mrad}$ and detector inner semiangle of $75 \mathrm{mrad}$ were employed with a pixel size of $0.094 \mathrm{~nm}$. Electron energy loss spectroscopy was used to determine a specimen thickness of $25 \pm 5 \mathrm{~nm}$ in the region of the $\{311\}$ defect using the mean-free path of total inelastic scattering [14].

The Tersoff 3 potential [15] has been shown to give a good description of both point defects and Si structures different from perfect diamond [16,17], as well as to predict the formation of $\{311\}$ defects from self-interstitial and bond defect rows [13]. Here we use the Tersoff 3 potential to perform MD simulations showing the spontaneous formation of $\{311\}$ defects. The outcome of the MD simulations serves to structurally characterize these defects. We have chosen the $X-, Y$ - and $Z$-cell axes to lie along the [ $2 \overline{3} \overline{3}],[311]$, and [0 $\overline{1} 1]$ directions, respectively, due to the particular orientation of $\{311\}$ defects. The smallest orthorhombic unit cell [10] that can reproduce the diamond $\mathrm{Si}$ lattice with these particular axis orientations contains 44 atoms and is of size $a \sqrt{11} / \sqrt{2} \times a \sqrt{11} \times a / \sqrt{2}, a$ being the lattice constant of diamond-structure $\mathrm{Si}$ $(5.43 \AA)$. We have used $6 \times 3 \times 6$ unit cells, introducing eight interstitial rows and seven bond defect rows (the number of interstitial rows was motivated by the experimental structure), for a total of 4800 atoms. We applied periodic boundary conditions along the $X$ and $Z$ axes, and free boundary conditions along the $Y$ axis to allow for the volume relaxation of the MD cell (only expansion along the [311] direction is expected due to the particular displacement vector of the $\{311\}$ defect). We have carried out annealing simulations at $1400 \mathrm{~K}$, high enough to accelerate system dynamics but below the melting point of diamond $\mathrm{Si}$. During the structural transformation energy is freed in the form of latent heat. If no special care is taken to eliminate such excess heat (that in real Si would dissipate through the lattice), it remains within the MD cell due to the periodic boundary conditions. To avoid such an artificial temperature increase, atom velocities were rescaled every 1000 time steps to maintain a constant temperature in the cell. We have used the fourth order Gear predictorcorrector algorithm to integrate the equations of motion

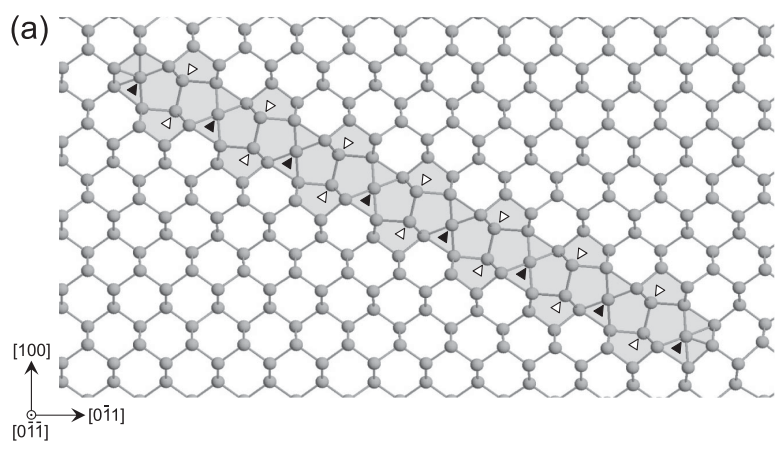

(b)

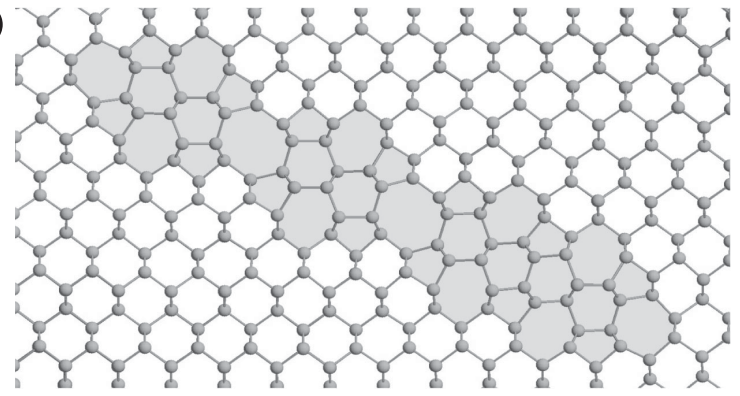

FIG. 1. $X Y$ snapshots of the MD simulation cell: (a) initial configuration, where solid and open triangles indicate Si interstitials and bond defect atoms, respectively; and (b) after 2 ns annealing at $1400 \mathrm{~K}$. Defect areas are shadowed for the sake of clarity.

[18]. Figure 1 shows the initial and final system configurations in one of the MD simulations. After $2 \mathrm{~ns}$, the defect formed by seven bond defect rows and eight interstitial rows has spontaneously transformed into a $\{311\}$ defect. Its structure, from left to right, is EIOIOIIE, where $E, I$, and $O$ refer to the structural unit types: $E$ units, which are seven-member rings at the end of the defect, acting as an interface between the defect and the bulk crystal; I units, which are two rods of hexagonal Si containing the interstitial $\langle 110\rangle$ chains; and $O$ units, which are eight-member rings with no excess or deficit of atoms compared to the bulk crystal [8].

Experimental images were obtained in the [0 $\overline{1}]$ direction and oriented such that the $X$ and $Y$ axes were parallel to [011] and [100], respectively, as indicated in the inset of Fig. 2(b). Atomic column positions $\mathbf{r}=(x, y)$ were determined by fitting two-dimensional Gaussians to each peak position in the unprocessed HAADF STEM image. The $x$ and $y$ standard deviations ( $\sigma_{x}$ and $\sigma_{y}$, respectively) of the Gaussian fit were taken as the uncertainty in the experimental peak positions. The MD positions of the atomic columns projected in $[0 \overline{1} \overline{1}]$ were calculated by averaging the $x$ and $y$ coordinates of atoms on each column in the modeled structure. Experimental peak positions were aligned to the theoretical positions using the Procrustes transformation, which optimally aligns two data sets using a rigid rotation, translation, and scaling [19]. In this case, 260 data points, which included peak positions of 160 columns associated with the bulk $\mathrm{Si}$ and 100 columns 
(a)
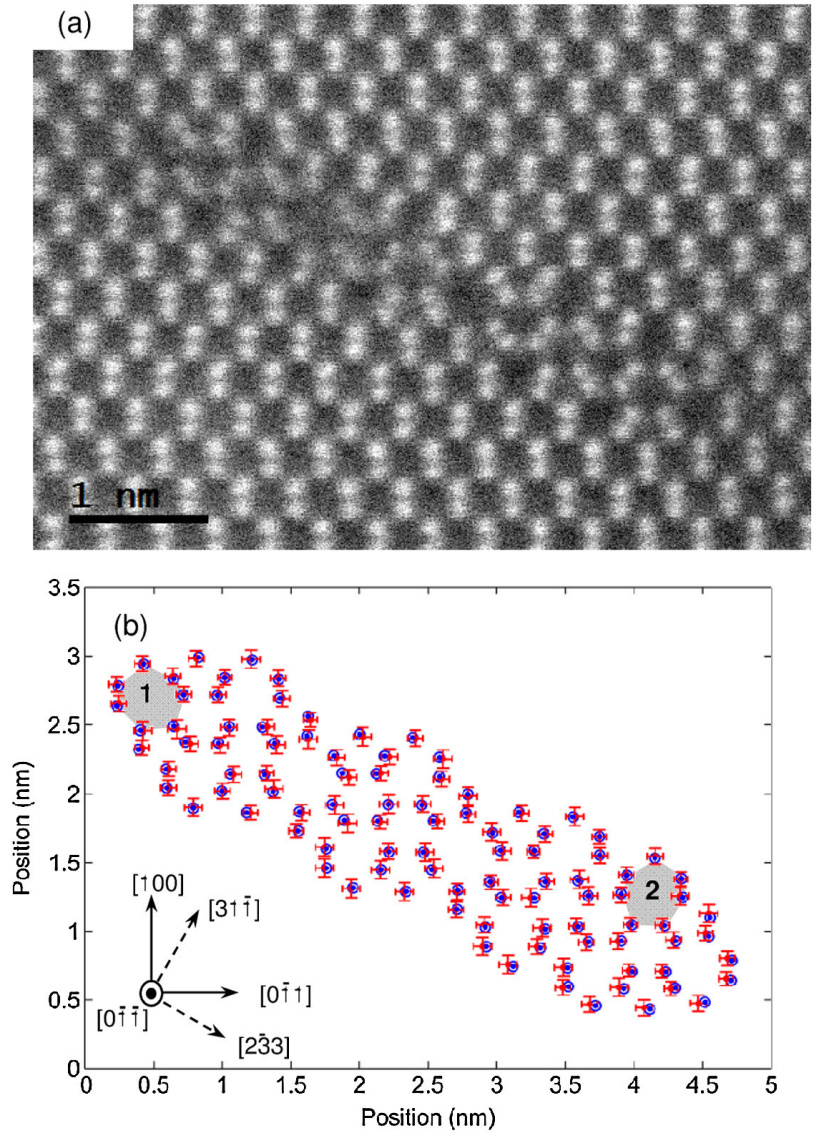

FIG. 2 (color online). (a) Unprocessed HAADF STEM image of the extended $\{311\}$ defect used to determine peak positions. (b) Structural units associated with the defect measured from the experimental column positions (red, filled squares) and aligned to theoretical column positions (blue, dotted circles). Error bars on experimental positions represent $\sigma_{x}$ and $\sigma_{y}$ of the Gaussian fit. The crystallographic coordinate system used in this Letter is shown as an inset in (b), with dashed axes indicating directions parallel and perpendicular to the defect. All experimental points are plotted with error bars, though they may appear within the plot symbols.

associated with the $\{311\}$ defect structure, were used to determine the optimal alignment.

Figure 2(a) presents the unprocessed HAADF STEM image used for experimental analysis. The aligned experimental and theoretical peak positions belonging to the $\{311\}$ defect are shown in Fig. 2(b). The defect structure, from left to right, is EIOIOIIE, in agreement with the model. Comparison of the absolute position of the experimental and theoretical column positions within the defect (100 columns) shows that all positions agree within $\pm 0.05 \mathrm{~nm}$, which is the average uncertainty in the experimental position and within 95\% confidence; agreement for the 160 columns in the bulk is consistent with that of the columns in the defect.

Detailed analysis of the experimental and modeled structures show that the shape of the individual rings that
TABLE I. Experimental and theoretical values for the lengths of the long and short sides, averaged over all rings.

\begin{tabular}{lcc}
\hline \hline & Long side $(\mathrm{nm})$ & Short side $(\mathrm{nm})$ \\
\hline Experiment & $2.40 \pm 0.09$ & $1.37 \pm 0.06$ \\
Theory & $2.36 \pm 0.02$ & $1.37 \pm 0.02$ \\
\hline \hline
\end{tabular}

make up the defect varies along its length. On average, the different five-, six-, seven-, and eight-member rings have two short sides and three to six long sides, with lengths as shown in Table I. In particular, the seven-member rings show two distinct shapes. The rings in the $E$ units are compressed along [100] compared to the rings in the I units. Figures 3(a) and 3(d) present seven-member rings from an $E$ and $I$ unit, as indicated by shaded regions numbered 1 and 2, respectively, in Fig. 2(b). The side lengths have the same trend and magnitude within uncertainty, as shown in Figs. 3(b) and 3(e). However, the trend in inner angles varies between the rings, as shown in Figs. 3(c) and 3(f): the third inner angle of the $E$ unit ring is smaller than the preceding inner angle, while the opposite is true of the $I$ unit ring. The same trends are observed in the rings in the
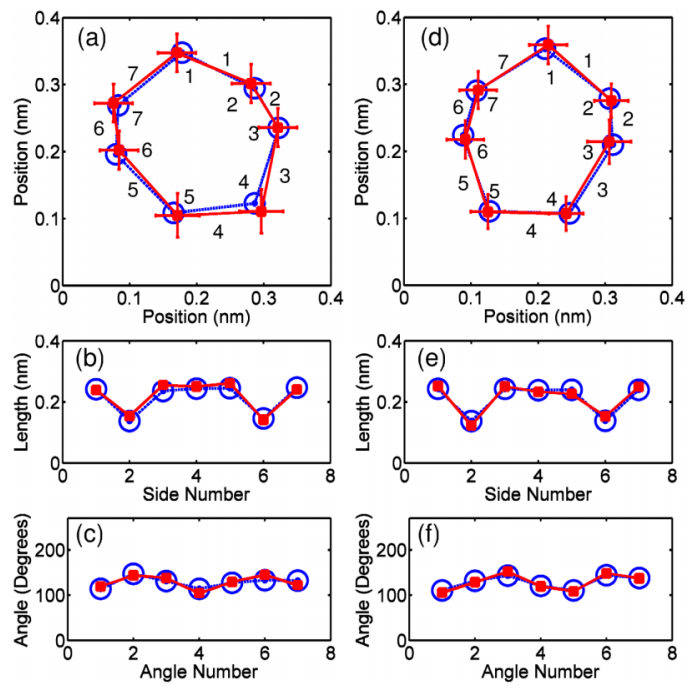

FIG. 3 (color online). Detailed structural comparisons of the seven-member rings indicated by shaded regions numbered 1 and 2 (respectively) in Fig. 2(b). (a)-(c) The structural comparison, side length, and interior angles (respectively) corresponding to the ring indicated by 1 in Fig. 2(b). (d)-(f) The structural comparison, side length, and interior angles (respectively) corresponding to the ring indicated by 2 in Fig. 2(b). Side and interior angle numbers are indicated on the outside and inside (respectively) of the rings shown in (a) and (d). Plot symbols are the same as those used in Fig. 2(b); solid red and dashed blue lines connecting experimental and theoretical data points (respectively) have been added as a guide for the eye. All experimental points are plotted with error bars, though they may appear within the plot symbols. Detailed structural, side length, and interior angle comparisons for all 34 rings are presented in the corresponding Supplemental Material [20]. 
two $E$ units and eight $I$ units. The subtle changes in side lengths and inner angle observed here were assessed due to the high quality of the experimental data and the detailed, precise, and accurate analysis of the projected column positions. Considering all 34 rings (representing 133 unique side lengths and 204 unique interior angles), the mean difference in side length is $0 \pm 0.02 \mathrm{~nm}$ and the mean difference in interior angle is $0^{\circ} \pm 6.49^{\circ}$. All experimental side lengths and interior angles agree with the corresponding theoretical lengths and angles within $95 \%$ confidence. Detailed structural, side length, and interior angle comparisons for all 34 rings are presented in the corresponding Supplemental Material [20].

Having determined the structure (EIOIOIIE) and positions of the columns within the $\{311\}$ defect, we can estimate the density of Si interstitials on $\{311\}$ planes. Following the approach of Takeda [6], the density of Si interstitials can be calculated as $4 / 6 \times 8 /\left(a^{2} \sqrt{11}\right)$ or $5.5 \times 10^{14} \mathrm{~cm}^{-2}$. This value is of the same order of magnitude as the value determined in Takeda's experiment $\left(5.1 \times 10^{14} \mathrm{~cm}^{-2}\right)$ [6] and agrees with the density calculated in the MD model.

In this Letter we have demonstrated that unprocessed HAADF STEM images can be used to achieve the subångstrom precision and accuracy required for quantitative comparison with calculated structures. The straightforward approach used here to quantifying the images is widely applicable, as it does not rely on knowledge of the defect chemistry, use of periodicity or averaging, or image processing methods. This offers a significant advantage over HRTEM-based methods, where the complex and often nonintuitive effects of the contrast transfer function can complicate interpretation and hence require extensive image simulation or phase reconstruction methods [3,21]. This approach to HAADF STEM image analysis has potential in the quantitative structural analysis of defects and interfaces, particularly in the case of complex or unknown structures.

Considering the particular example of an extended $\{311\}$ defect in $\mathrm{Si}$, we have demonstrated agreement between experiment and theoretical column positions of better than $\pm 0.05 \mathrm{~nm}$ for $100+$ atomic columns in a region larger than $15 \mathrm{~nm}^{2}$. We have shown that, although the $\{311\}$ defect is broadly made up of five-, six-, seven-, and eight-member rings, the shape of rings varies as a function of position in the defect, with unique side length and interior angle trends based on position. Assessment of these subtle changes required quantitative analysis of high-quality HAADF STEM images. The experimental results are well matched by theoretical findings and demonstrate the validity of the MD simulation in accurately and precisely predicting the structure of the extended defects.

K.J.D. received partial support from an Ontario Ministry of Economic Development and Innovation postdoctoral fellowship. G. A. B. is grateful to NSERC for a Discovery Accelerator Grant partially supporting this work. The experimental microscopy work was carried out at the Canadian Centre for Electron Microscopy, a National Facility supported by NSERC and McMaster University. L. A. M. is grateful to the Spanish Government for providing funding under Project No. TEC2011-27701.

*dudeckkj@mcmaster.ca

${ }^{\dagger}$ Corresponding author. gbotton@mcmaster.ca

[1] International Technology Roadmap for Semiconductors, http://public.itrs.net/.

[2] G. B. Winkelman, C. Dwyer, C. Marsh, T. S. Hudson, D. Nguyen-Manh, M. Döblinger, and D. J.H. Cockayne, Mater. Sci. Eng. A 422, 77 (2006).

[3] K. J. Dudeck, N. A. Benedek, M. W. Finnis, and D. J.H. Cockayne, Phys. Rev. B 81, 134109 (2010).

[4] D. J. Eaglesham, P. A. Stolk, H. J. Gossmann, and J. M. Poate, Appl. Phys. Lett. 65, 2305 (1994).

[5] P. Stolk, H.-J. Gossmann, D. Eaglesham, D. Jacobson, C. Rafferty, G. Gilmer, M. Jaraiz, J. Poate, H. Luftman, and T. Haynes, J. Appl. Phys. 81, 6031 (1997).

[6] S. Takeda, Jpn. J. Appl. Phys., Part 2 30, L639 (1991).

[7] S. Takeda, M. Kohyama, and K. Ibe, Philos. Mag. A 70, 287 (1994).

[8] M. Kohyama and S. Takeda, Phys. Rev. B 46, 12305 (1992).

[9] M. Kohyama and S. Takeda, Phys. Rev. B 51, 13111 (1995).

[10] J. Kim, J. W. Wilkins, F. S. Khan, and A. Canning, Phys. Rev. B 55, 16186 (1997).

[11] P. Alippi and L. Colombo, Phys. Rev. B 62, 1815 (2000).

[12] J. P. Goss, T. A. G. Eberlein, R. Jones, N. Pinho, A. T. Blumenau, T. Frauenheim, P. R. Briddon, and S. Oberg, J. Phys. Condens. Matter 14, 12843 (2002).

[13] L. A. Marqués, L. Pelaz, I. Santos, P. Lopez, and M. Aboy, Phys. Rev. B 78, 193201 (2008).

[14] R. F. Egerton, Electron Energy-Loss Spectroscopy in the Electron Microscopy (Plenum, New York, 1996), 2nd ed.

[15] J. Tersoff, Phys. Rev. B 39, 5566 (1989).

[16] S. Munetoh, K. Moriguchi, K. Kamei, A. Shintani, and T. Motooka, Phys. Rev. Lett. 86, 4879 (2001).

[17] L. A. Marqués, L. Pelaz, M. Aboy, L. Enríquez, and J. Barbolla, Phys. Rev. Lett. 91, 135504 (2003).

[18] C. W. Gear, Numerical Initial Value Problems in Ordinary Differential Equations (Prentice-Hall, Englewood Cliffs, NJ, 1971).

[19] D. Kendall, Stat. Sci. 4, 87 (1989).

[20] See Supplemental Material at http://link.aps.org/ supplemental/10.1103/PhysRevLett.110.166102 for detailed structural, side length, and interior angle comparisons for all 34 rings.

[21] G. Möbus, R. Schweinfest, T. Gemming, T. Wagner, and M. Rühle, J. Microsc. 190, 109 (1998). 